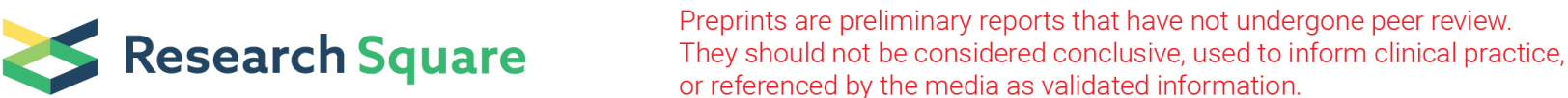

\section{Factors associated with changing behaviour for arboviruses prevention: evidence from a quasi- experimental intervention study using mobile devices.}

Silvana Santos ( $\nabla$ silvanaipe@gmail.com )

State University of Paraiba https://orcid.org/0000-0002-5252-0206

Roberta Smania-Marques

Universidade Estadual da Paraiba

Victor Alves Albino

Universidade Estadual da Paraiba

Izabelly Dutra Fernandes

Universidade Estadual da Paraiba

Francisco Fernandes Abel Mangueira

Universidade Estadual da Paraiba

Ricardo Olinda

Universidade Estadual da Paraiba

Carol S Bond

University of Wolverhampton

David Matheson

University of Wolverhampton

John Traxler

University of Wolverhampton

Research article

Keywords: Arboviruses, Health Education, Behaviour Change

Posted Date: March 18th, 2020

DOl: https://doi.org/10.21203/rs.3.rs-17586/v1

License: (c) (1) This work is licensed under a Creative Commons Attribution 4.0 International License.

Read Full License 


\section{Abstract}

Background: Dengue was reintroduced in Brazil in the 80's. Since 2010, it is estimated that there have been over a million cases of dengue per year, leading to hundreds of deaths. Community health education is one of the main objectives of vector control policies.

Objective: Here we compare perceptions and behaviours before and after an educational intervention based on behavioural change theories (BCT) of a group of Endemic Disease Control Agents (ACEs) with a group of college students of Campina Grande city, Paraiba state, Brazil.

Methods: Using a distance-learning platform adapted for mobile devices, the intervention consisted of tasks or missions that were presented through short videos with people performing the desired target behaviour. To demonstrate the accomplishment of the tasks, participants produced videos and shared on social networks. A questionnaire was completed before and after the intervention by 58 participants, 31 students and 27 ACEs.

Results: Most of the participants were female (83.9\% and $72.2 \%$ of college students and ACEs, respectively); $27.8 \%$ of ACEs attended higher education and $70.4 \%$ were married contrasting with $9.8 \%$ of the students. Among college students, a significant difference in the chi-square test was found for all tentarget behaviours after the intervention. ANOVA analysis showed that ACEs have a higher perception of susceptibility and more fear of acquiring arboviruses than students do; and there was a correlation between facilitating behaviours with behaviour change.

Conclusion: Intervention based on BCT thus contributed to bring students' behaviour patterns closer to those of ACEs.

\section{Background}

Dengue was reintroduced in Brazil in the 80's [1-2]. Since 2010, it is estimated that there have been over a million cases of dengue per year, leading to hundreds of deaths [2]. From 2015, with the introduction of the Zika and Chikungunya viruses in the country, cases have been reported of children with congenital Zika syndrome or chronic sequelae [2-5]. Community health education is one of the main objectives of vector control policies, given that most of the breeding sites are located in households [6].

This paper describes the results of an educational intervention based on behavioural change theories aimed at preventing the spread of arboviruses. A distance-learning platform adapted for mobile devices was used to engage and mobilize the population in arboviral prevention actions. During the educational intervention, participants learned to inspect their homes, keep water reservoirs capped, separate recyclables to donate to pickers, put screens on windows, and implement procedures to identify where mosquitoes might breed and hence make clean-up efforts. The content was taught through short videos with examples of people performing the desired target behaviour. This study that corresponds to the 
second phase of the "Impact of mobile learning on the prevention and management of complications caused by arbovirus (Zika, Dengue, Chikungunya) - ZIKAMOB" project, founded by British Council.

At this phase, the intervention aimed to train the population to perform actions, which are carried out by the Endemic Disease Control Agents (Agentes de Combate às Endemias - ACEs). These professionals are responsible for inspecting public and private properties to control vector mosquito populations, since in these places there is a higher incidence of Aedes outbreaks [7]. ACEs should also guide and disseminate information to communities to prevent diseases caused by mosquito vectors [7-9]. During visits, ACEs survey water deposits to identify outbreaks and explain to the population the importance of keeping these containers clean, avoiding the accumulation of water and solid waste that can serve as breeding sites. Having identified the presence of larvae or mosquitoes in a neighbourhood, ACE then uses insecticides, including cold spatial fogging in the area around where the larvae or mosquitoes have been found, thereby reducing the vector population [10-11].

In this paper, we compare perceptions and behaviours before and after an educational intervention based on behavioural change theories of a group of ACEs with a group of college students. ACEs already have the target behaviours that every citizen or population must develop in order to reduce vector mosquito infestation levels; college students may or may not have these behaviours. Our working hypothesis considers that educational intervention should approximate the pattern of students' perceptions and behaviours to those of ACEs.

In short, we intend to answer the following guiding questions:

1 - What differences exist in the perceptions and behaviours of ACEs and under-graduate students before and after the intervention?

2 - What factors are associated with behavioural change? (sociodemographic characteristics, external factors such as risks and benefits, intrinsic factors such as motivation, self-efficacy, perceptions of susceptibility and severity, intention, among others).

3 - Which constructs of behavioural change theories explain the findings?

4 - How do the results contribute to the planning of future educational actions and public policies aimed at preventing arboviruses?

\section{Methods}

This is a quasi-experimental two-group intervention study, one composed of Endemic Disease Control Agents (ACEs) and one of college students. The study was conducted in the city of Campina Grande, being the second largest urban centre of Paraíba in north-eastern Brazil and currently has 355,000 inhabitants. In the city, there are about 350 ACEs linked to the Entomological Surveillance of the municipality. 
The study population included 100 students and 50 ACEs who signed up for a voluntary distance learning course which would use mobile devices. The course was disseminated through the university's website and in partnership with the Department of Health of the Municipality of Campina Grande, which selected 50 agents to participate in the course.

Participation was in the form of a scavenger hunt where a cash prize was offered to those who completed all activities, including the course evaluation. The project was initiated after approval by the Research Ethics Committee of the State University of Paraíba (Protocol CAAE 67429517.5.0000.5187) and due consent of the participants gained by their signing the informed consent form.

For the purpose of statistical analysis, only participants who completed all of the elements of the programme, answering both questionnaires, before and after the intervention, and performing all activities of the intervention. Participants who did not complete these steps or who skipped the course were excluded. The final sample consisted of 58 participants, 31 students and 27 ACEs.

\section{Intervention - Theoretical background}

The World Health Organization (WHO) published in 2012 a document entitled "Health Education: Theoretical Concepts, Effective Strategies and Core Competencies" which outlines the main theories of behavioural change and those that can be used in educational interventions [12]. The WHO highlighted nine behavioural change theories that have been widely used in health interventions; three were selected whose constructs were studied in this investigation. The Theory of Rational Action (TRA) considers that knowledge contributes to changing attitudes and beliefs, which are essential for behavioural change [12]. The Health Belief Model (HBM) advocates that decision-making depends on the perception of susceptibility, disease severity, benefits and barriers associated with behaviour [12]. Later, the model added the concept of self-efficacy. Social Cognitive Theory (SCT) describes three main factors that affect a person's likelihood of changing health behaviour: self-efficacy, goals, and outcome expectations. Learning target behaviours can be facilitated by observing people performing the same [12].

Changing the frequency with which a person performs a particular behaviour requires a change in at least one of the following observables: ability, motivation, or opportunity to engage in the activity [13-14]. Ability refers to the physical and psychological condition to perform a behaviour. Motivation involves all processes that energize and direct behaviour, including not only goals, plans, and beliefs, but also "automatic" processes that involve emotions, habits, and impulses. Opportunity involves all factors external to an individual that can influence engagement in an activity, ranging from the physical environments in which people spend time to the social and cultural environment that determines how we perceive and think about actions. It includes reducing the distance between intention and action. Therefore, to maximize the potential benefit of behaviour change interventions, it is important to understand that capacity, motivation, and opportunity vary depending on specific behaviours, target populations, and social and environmental contexts [13-14]. 
Experience in performing a behaviour can make it habitual, so that intention and motivation over time become less important in determining individual performance. In order to foster change, it is necessary to show people performing the target behaviour that one wants to establish in the population, and transmitting personalized, interactive and accessible information. However, it is not necessary to link the preliminary or previous change in attitudes to the subsequent change in behaviour, as both may occur concomitantly [15-17]. Familiarity, observation of others performing the desired behaviour, and social norms are predictors of behaviour change. Table 1 summarizes the constructs of behaviour change theories selected for study in this investigation containing a brief description of the theory and concepts, and some questions for reflection.

\section{Intervention - description and assessment tools}

Using a distance-learning platform adapted for mobile devices, the intervention consisted of tasks or missions that were presented through short videos with people performing the desired target behaviour. To demonstrate the accomplishment of the tasks, participants produced audio-visual content (videos) and shared on social networks (Facebook). The posts had specific hashtags such that it was possible to track the post and interactions on social networks. In addition, the participant also included the link to their publication on the virtual platform. There were ten tasks to be performed in the 40-hour course, held between October and December 2018, with two face-to-face meetings, one to explain the objectives and operation of the platform and the other to reward participants. This strategy was previously described and tested by Mangueira et al (2019) [18].

During the course, the content of the missions was evaluated by the ACEs and students to identify possible errors in inspection procedures or elimination of mosquito breeding sites. Thus, the participants also answered some questions to evaluate the quality and relevance of each mission so that the content could be reproduced with students and teachers of Basic Education in the next stage of the project.

The assessment of perceptions and behaviours before and after the intervention was performed by applying a self-reported questionnaire containing questions with binary yes and no answers. Positive responses indicated that the participant had perception and preventive behaviour, or less risk of acquiring arboviruses, and they were worth 1 to 3 points; negative answers, or those that were not applicable to the participant's situation, were worth zero points.

Each of the behaviours target prevention and breeding elimination was considered a dependent categorical variable. The sum of the score for the ten questions related to prevention actions led to the "Prevention Score", which could range from 0 to 10 points; which could be added to the score of the five answers regarding breeder elimination ("Breeding Elimination Score") thus giving rise to the "Target Behaviour Score" (0 to 15 points).

Independent variables were grouped into the following subgroups: 
Sociodemographic variables such as gender, age, marital status, income, occupation and whether the participant had children.

2) Variables related to environmental risk that encompassed conditions outside the participant's home such as the existence of vacant land or abandoned houses, offer of garbage collection services, running water and entomological surveillance. The sum of the score of the answers gives rise to the "Environmental Risk Score", ranging from 0 to 5 points;

3) Home risk corresponds to conditions that may or may not facilitate the proliferation of mosquitoes in the participant's home. For example, if the person lives in rented accommodation, they are likely to be less interested in window screens or home improvements. Roofless houses with water tanks, water storage containers, gardens and plants are more likely to have breeding grounds. Positive statements were scored and the higher the value of this score, the greater the risk of having breeding grounds at the participant's residence. Thus, the "Home Risk Score" ranges from zero to ten points. The sum of the two previous scores gave rise to the "Environmental and Household Risk Score", ranging from 0 to 15 points.

4) Constructs of Behaviour Change Theories: 25 assertions were proposed with which the participant could agree or not; being assigned one to three points for positive responses that revealed knowledge, perceptions, attitudes and beliefs that favour behaviour change. The sum of the scores gave rise to different scores: "Knowledge Score" (TRA) consisting of five assertions; "Self-efficacy and observation score" (SCT) that evaluated the constructs of self-efficacy, collective efficacy and observation; the "Facilitator Score" (SCT) which assessed familiarity, the existence of target behaviours prior to intervention, and behaviours that facilitate behaviour change. Finally, constructs of the Health Belief Theory ("Health Belief Score") (HBT), such as perception of susceptibility, severity, barriers and benefits associated with behaviour change, were analysed. All positive answers meant having a positive understanding or perception of the concept examined. The "Behaviour Change Theories Score" resulted from the sum of all scores and ranged from 0 to 25 points. This score allowed us to measure whether the participant changed his perceptions after the educational activities.

\section{Statistical analysis}

Descriptive statistics were used to describe the population profile and the frequency of each questionnaire response before and after the intervention. To assess whether there were differences in the median (categorical variables) or mean (normal distribution scores) values between the groups analysed; the Chi-square test, Fisher's exact test and ANOVA Kruskal Wallis and Friedman tests for independent and paired samples were used [19], considering the significance level of $5 \%(p$-value $<0.05)$. The reliability analysis of the instrument was performed using Cronbach's alpha. The analyses were performed with the aid of the R statistical software [20]. 
Secondly, multivariate analysis techniques were used, adjusting the Principal Component Analysis (PCA), whose eigenvalues were greater than one $(\lambda>1)$, as suggested by Kaiser (1960) [21], in order to identify a smaller number of variables. uncorrelated alternatives that somehow summarize the main information of the original variables. Subsequently, these main components were presented in Biplot graphs for individuals and variables with their respective confidence ellipses (with $95 \%$ reliability). Biplot is a method that represents two-dimensional multivariate data, where each observation is represented by the pair of scores of the first two main components, representing each group (students and agents) in their respective confidence ellipses.

\section{Results}

\section{Socioeconomic Profile, environmental and household risk}

Table 2 shows the results for the category of students and ACEs before and after the intervention regarding socio economic profile, environmental and household risk. Regarding gender, $83.9 \%$ and $72.2 \%$ of students and ACEs, respectively, were female, with no significant difference between them $(p=0.128)$. All college students were starting higher education and only $9.8 \%$ of them were married or had a stable union. In the ACEs group, $27.8 \%$ of them attended higher education and $70.4 \%$ were married. These differences between the groups were significant $(p<0.001)$. Regarding family income, about $75 \%$ of both students and ACEs live on two to six minimum wages (US\$ 500 up to US\$1,500).

About $60 \%$ of students and ACEs have some vacant land near their homes; 93.5 to $100 \%$ said they had running water and garbage collection at least twice a week; about $30 \%$ of participants have open sewers or streams near their homes. When asked if they were visited by an endemic control agent, there was a significant difference between the groups ( $p<0.001$ ), while $25 \%$ of the students replied positively, among the ACEs, this value was $100 \%$ since they themselves are responsible for performing this service (Table 1).

To verify the reliability of the students 'and ACEs' answers, the same questions regarding sociodemographic and environmental and household risk variables were used before and after the test; no significant difference between them for both groups. Therefore, the answers were reproduced as expected (Table 2). The analysis of Cronbach's alpha results considering 102 categorical variables used before and after the test showed a value of 0.842 , indicating that the instrument has good reliability and validation.

\section{Knowledge, Perceptions, and Constructs of Behaviour Change Theories}

ACEs are responsible for teaching arboviral prevention practices to the community, and they deal with the prospect of getting sick every day or the phenomenon of diseases such as zika, dengue and chikungunya. They therefore have more knowledge, attitudes and positive behaviours towards prevention than any other segments of society. In the Rational Theory (TRA) block, of the five statements that 
portrayed specific knowledge, in two there was a significant difference between the group of ACEs and college students. In fact, $96.3 \%$ ACEs and $82.3 \%$ of students knew that Aedes aegypti mosquito larvae are photophobic ( $p=0.017)$ and $98.1 \%$ of ACEs and $87.1 \%$ of students knew that it was necessary to perform the identification of mosquito larvae species for infestation counting $(p=0.026)$. More than $95 \%$ of both groups knew about mosquito habits and, in relation to reporting Health Surveillance, $100 \%$ answered affirmatively (Table 3).

In the block on the Cognitive Social Theory, the three statements that dealt with the belief of the individual, their family and community, are able to perform preventive activities. The totality of both groups answered affirmatively. Regarding the use of mobile phones and the behaviour of posting and viewing contents in social media, there was a significant difference between the two groups: $83.9 \%$ of ACEs and $75.8 \%$ of students $(p<0.01)$ (Table 3$)$.

Regarding the Health Belief Theory, only two had significant differences between ACEs and college students: $66.7 \%$ of ACEs and $48.4 \%$ of students reported having symptoms of some arbovirus ( $p=$ 0.047); and $98.1 \%$ of the ACEs and $85.5 \%$ of the students reported being afraid of acquiring these diseases. The ACEs, who work directly with this theme, are more afraid than students of acquiring arboviruses and the prevalence of these diseases is higher in the group of ACEs (Table 3). There was no difference regarding the perception of risk, severity, benefits and barriers between the two categories.

Comparison of data before and after the intervention for each category separately showed that there was behaviour change only in the student group (Table 3). Regarding the acquisition of knowledge, only one of the statements had significant difference with the intervention, which concerns the photophobia of the larvae $(p=0.043)$. In the blocks related to the constructs of the health belief theory, there was no difference due to the intervention. However, considering the facilitating behaviours that coincided with the behaviours to be modified with the intervention, there was a significant difference in four of the five variables analysed (Table 3 ).

\section{Target Behavioural Changes}

The educational activities aimed to modify ten target behaviours and, if the participant had found a breeding site, he should perform five actions to ensure the resolution of the problem according to the guidelines of the Environmental Surveillance. In the case of ACEs, it was expected that they already had these behaviours in their daily lives, and, in fact, there was a difference only in relation to the screening of windows and drains $(p=0.001)$ (Table 4$)$. For the group of college students, a significant difference in the chi-square test was found for all ten-target behaviours after the intervention. In the comparison between the two categories (students and ACEs), it was found that there was a significant difference between them for all target behaviours except for having a repellent plant pot or using repellents and tiling windows and drains (Table 4). 


\section{Comparison and correlation of scores}

Table 5 shows ANOVA analysis results comparing the scores before and after the intervention for the different groups and between them. No significant differences in environmental and household risk variables were observed, because they have not changed over time. In relation to the other scores, we found that there is a clear difference in relation to the constructs of the Health Belief Theory when comparing the ACEs and the students. In fact, ACEs have a higher perception of susceptibility and more fear of acquiring arboviruses than do the students. After the intervention, students feel safer because they are more knowledgeable and have taken more measures that are preventive. This change in the perception of susceptibility and severity did not occur in the ACEs group.

Regarding the results of Spearman's correlation analysis, we found that there is indeed a correlation between facilitating behaviours, i.e. the fact that the participant already has some of the behaviours before the intervention with the behaviour change; that is, which highlights the importance of the concept of familiarity and of performing the behaviour (Table 6). There was also a correlation between the health belief theory score and the dependent variables in the case of ACEs before and after the intervention; evidencing that the ACEs have not lost their fear of acquiring arboviruses. On the other hand, it was found that, in the case of students, there was correlation just before the intervention. This shows that students, in fact, changed their perception of susceptibility and severity with the intervention (Table 6).

\section{Multivariate analysis}

Figure 1 shows the Biplot plot from Principal Component Analysis (PCA) showing different patterns for the two groups analysed before and after the intervention. When analysing the dispersion ellipses, the ACEs group has less variability in relation to sociodemographic characteristics, perceptions and behaviours than college students. The two groups have quite different standards regarding arbovirus prevention, as expected. With the intervention, it was found that students' appropriate knowledge, perceptions and behaviours in such a way as to overlap with the ACEs. Intervention based on behaviour change theories thus contributed to bring students' behaviour patterns closer to those of ACEs.

\section{Discussion}

This study showed that educational interventions based on behaviour change theory and $\mathrm{m}$-health can contribute to engage and mobilize the population in arbovirus prevention actions, corroborating the 
findings of Mangueira et. al. [18]. In the literature, most studies describe perceptions and knowledge about arbovirus prevention [22-29] with few reports of educational interventions using ICTs.

As already pointed out in the literature, the main predictor of behaviour change is already having previously targeted behaviour or related behaviours [12-16]. Mangueira et al (2019) [18] found that military police officers who are trained to keep spaces clean and organized inspect their homes to identify mosquito breeding sites more often than college students. The present work, however, shows that stimulating domestic activities among young people can contribute to them developing arboviral prevention behaviours as adults.

In the present work, the educational intervention did not contribute to increase the students' fear; on the contrary, they felt safer and the perception of susceptibility decreased over time. The fact that students perform preventive actions may have contributed to reduce their fear of acquiring the disease. In fact, data on the mortality rates of arboviruses or information that made students reflect on the severity of these diseases were not included in educational activities. Other published studies have shown that some risk groups, such as pregnant women, have a different perception of susceptibility to arboviruses [30].

Whether or not living at home and in a region most at risk for mosquito breeding sites did not imply more behaviours that are preventive. In fact, in the ACEs group, participants with higher household risk showed more target behaviours in relation to the elimination of mosquito breeding sites. In the literature, some studies have shown that the perception of those residing in risk areas is different from those in areas with lower risk [31], and there are differences between those who live in urban and rural areas [28]. The findings of the present work give rise to some working hypotheses, which can be tested in further studies with larger samples.

\section{Declarations}

\section{Author's contributions}

SS: study conception, study design, acquisition of data, analysis and interpretation of data, and drafting the manuscript. RSM, FFAM, VAA and IDF: acquisition of data, analysis and interpretation of data. RO: statistical analysis. CSB, DM and JT: drafting the manuscript and final revision. All authors read and approved the final manuscript.

\section{Consent for publication}

Not applicable.

\section{Availability of data and material}


The datasets used and/or analysed during the current study are available from the corresponding author on reasonable request.

\section{Competing interests}

The authors declare that they have no competing interests.

\section{Funding}

The current study was funded by Fundação de Apoio à Pesquisa do Estado da Paraíba (FAPESQ - Edital Zika Virus 02/2017), CAPES and British Council 274560199. The role of the funding body: the design of the study; collection, analysis, and interpretation of data; and in writing the manuscript.

\section{Ethics approval and consent to participate}

This research was approved by the Research Ethics Committee of Paraiba State University (UEPB) under protocol CAAE 67429517.5.0000.5187 and was in accordance with the principles of Resolution 466/12 of the Brazilian National Health Council.

\section{Conclusions}

The course we provided encompassed not only theories of behavioural change in its design and implementation; it was also presented in a format easily accessible to our participants. Effectively, we reduced the distance between intention and action. It stimulated motivation by means of interim tasks to be accomplished and displayed on social media, thereby stimulating a sense of group identity and perhaps even some sense of competition whereby participants would not wish to be seen not to have performed the set tasks. And then there was also the cash prize for those who completed all the tasks. These factors came together to bring the students' behaviour pattern in combating vector mosquitoes closer to that of the ACEs and also giving the students a greater sense of agency in respect of prevention of arbovirus infection.

In sum, we have presented a model for effectuating behavioural change perhaps applicable in other settings.

\section{References}

1. Villabona-Arenas, C. J., de Oliveira, J. L., Capra, C. de S., Balarini, K., Loureiro, M., Fonseca, C. R. T. P., ... Zanotto, P. M. de A. (2014). Detection of four dengue serotypes suggests rise in hyperendemicity in urban centers of Brazil. PLoS neglected tropical diseases, 8(2), e2620.

https://doi.org/10.1371/journal.pntd.0002620

2. Zanotto, P. M. de A., \& Leite, L. C. de C. (2018). The Challenges Imposed by Dengue, Zika, and Chikungunya to Brazil. Frontiers in immunology, 9, 1964. https://doi.org/10.3389/fimmu.2018.01964 
3. Lima-Camara, T. N. (2016). Emerging arboviruses and public health challenges in Brazil. Revista de saude publica, 50. https://doi.org/10.1590/S1518-8787.2016050006791

4. Nunes, M. R. T., Faria, N. R., de Vasconcelos, J. M., Golding, N., Kraemer, M. U. G., de Oliveira, L. F., ... Vasconcelos, P. F. da C. (2015). Emergence and potential for spread of Chikungunya virus in Brazil. BMC medicine, 13, 102. https://doi.org/10.1186/s12916-015-0348-x

5. Lowe, R., Barcellos, C., Brasil, P., Cruz, O. G., Honório, N. A., Kuper, H., \& Carvalho, M. S. (2018). The Zika Virus Epidemic in Brazil: From Discovery to Future Implications. International journal of environmental research and public health, 15(1). https://doi.org/10.3390/ijerph15010096

6. Lima-Camara, T. N., Urbinatti, P. R., \& Chiaravalloti-Neto, F. (2016). Finding Aedes aegypti in a natural breeding site in an urban zone, Sao Paulo, Southeastern Brazil. Revista de saude publica, 50, 3. https://doi.org/10.1590/S1518-8787.2016050006245

7. Guida, H. F. S., de Souza, K. R., dos Santos, M. B. M., da Silva, S. M. C. L., \& Silva, V. P. (2012). The relationship between health and work of FUNASA's endemic diseases combat agents. Saúde $e$ Sociedade. https://doi.org/10.1590/s0104-12902012000400006

8. Fraga, L. dos S., dos Santos Fraga, L., \& Monteiro, S. (2014). We are information dealers.educational practices of endemic diseases' battling agents at a service of zoonoses control in Belo Horizonte, Brazil. Saúde e Sociedade. https://doi.org/10.1590/s0104-12902014000300021

9. Libanio, K. R., Favoreto, C. A. O., \& Pinheiro, R. (2014). Analysis of the integration between Environmental Surveillance in dengue control and the Family Health Strategy: Impact on knowledge and practice of community health workers. Physis: Revista de Saúde Coletiva. https://doi.org/10.1590/s0103-73312014000100009

10. Silva, I. B. da, da Silva, I. B., Mallmann, D. G., \& de Vasconcelos, E. M. R. (2015). Strategies to combat dengue through health education: an integrative review. Saúde (Santa Maria). https://doi.org/10.5902/2236583410955

11. Pessoa, J. P. de M., de Morais Pessoa, J. P., de Oliveira, E. S. F., Teixeira, R. A. G., Lemos, C. L. S., \& de Barros, N. F. (2016). Control of dengue: consensus views of Endemic Disease Control Agents and Community Health Agents on their integrated action. Ciência \& Saúde Coletiva. https://doi.org/10.1590/1413-81232015218.05462016

12. World Health Organization. (2012). Health Education: Theoretical Concepts, Effective Strategies and Core Competencies. Retrieved from https://books.google.com/books/about/Health_Education.html? $\mathrm{hl}=\& \mathrm{id}=\mathrm{fP} 8 \mathrm{uLgEACAAJ}$

13. Michie, S., Atkins, L., \& West, R. (2014). The Behaviour Change Wheel: A Guide to Designing Interventions. Retrieved from https://books.google.com/books/about/The_Behaviour_Change_Wheel.html?hl=\&id=1TGIrgEACAAJ

14. West, R., Evans, A., \& Michie, S. (2011). Behavior change techniques used in group-based behavioral support by the English stop-smoking services and preliminary assessment of association with shortterm quit outcomes. Nicotine \& tobacco research: official journal of the Society for Research on Nicotine and Tobacco, 13(12), 1316-1320. https://doi.org/10.1093/ntr/ntr120 
15. Glanz, K., \& Bishop, D. B. (2010). The role of behavioral science theory in development and implementation of public health interventions. Annual review of public health, 31, 399-418. https://doi.org/10.1146/annurev.publhealth.012809.103604

16. Michie, S., \& Abraham, C. (2004). Interventions to change health behaviours: evidence-based or evidence-inspired? Psychology \& Health. https://doi.org/10.1080/0887044031000141199

17. Noar, S. M., \& Zimmerman, R. S. (2005). Health Behavior Theory and cumulative knowledge regarding health behaviors: are we moving in the right direction? Health education research, 20(3), 275-290. https://doi.org/10.1093/her/cyg113

18. Abel Mangueira, F. F., Smania-Marques, R., Dutra Fernandes, I., Alves Albino, V., Olinda, R., Acácia Santos-Silva, T., ... Santos, S. (2019). The prevention of arboviral diseases using mobile devices: a preliminary study of the attitudes and behaviour change produced by educational interventions. Tropical medicine \& international health: TM \& IH, 24(12), 1411-1426.

https://doi.org/10.1111/tmi.13316

19. Siegel, A. (2011). Practical Business Statistics. Academic Press. Retrieved from https://play.google.com/store/books/details?id=1ICwX1uWWhoC

20. R: The R Project for Statistical Computing. (n.d.). Retrieved February 25, 2020, from https://www.rproject.org/

21. Kaiser, H. F. (1960). The Application of Electronic Computers to Factor Analysis. Educational and Psychological Measurement. https://doi.org/10.1177/001316446002000116

22. Darrow, W., Bhatt, C., Rene, C., \& Thomas, L. (2018). Zika Virus Awareness and Prevention Practices Among University Students in Miami: Fall 2016. Health education \& behavior: the official publication of the Society for Public Health Education, 45(6), 967-976. https://doi.org/10.1177/1090198118760687

23. Feldstein, L. R., Rowhani-Rahbar, A., Staples, J. E., Halloran, M. E., \& Ellis, E. M. (2018). An Assessment of Household and Individual-Level Mosquito Prevention Methods during the Chikungunya Virus Outbreak in the United States Virgin Islands, 2014-2015. The American journal of tropical medicine and hygiene, 98(3), 845-848. https://doi.org/10.4269/ajtmh.17-0799

24. Nyunt, M. H., Aye, K. M., Kyaw, M. P., Wai, K. T., Oo, T., Than, A., ... San, K. K. (2015). Evaluation of the behaviour change communication and community mobilization activities in Myanmar artemisinin resistance containment zones. Malaria journal, 14, 522. https://doi.org/10.1186/s12936-015-1047-y

25. Plaster, A. N., Painter, J. E., Tjersland, D. H., \& Jacobsen, K. H. (2018). University Students' Knowledge, Attitudes, and Sources of Information About Zika Virus. Journal of Community Health. https://doi.org/10.1007/s10900-017-0463-z

26. Samuel, G., DiBartolo-Cordovano, R., Taj, I., Merriam, A., Lopez, J. M., Torres, C., ... Thakur, K. T. (2018). A survey of the knowledge, attitudes and practices on Zika virus in new York City. BMC public health, 18(1), 98. https://doi.org/10.1186/s12889-017-4991-3

27. Storey, J. D., Babalola, S. O., Ricotta, E. E., Fox, K. A., Toso, M., Lewicky, N., \& Koenker, H. (2018). Associations between ideational variables and bed net use in Madagascar, Mali, and Nigeria. BMC 
public health, 18(1), 484. https://doi.org/10.1186/s12889-018-5372-2

28. Ramaiah, R., \& Jayarama, S. (2018). Awareness and practices related to dengue fever among rural high school students: a cross sectional study. International Journal Of Community Medicine And Public Health. https://doi.org/10.18203/2394-6040.ijcmph20181207

29. Wong, L. P., \& AbuBakar, S. (2013). Health beliefs and practices related to dengue fever: a focus group study. PLoS neglected tropical diseases, 7(7), e2310.

https://doi.org/10.1371/journal.pntd.0002310

30. Khun, S., \& Manderson, L. (2007). Community and school-based health education for dengue control in rural Cambodia: a process evaluation. PLoS neglected tropical diseases, 1(3), e143. https://doi.org/10.1371/journal.pntd.0000143

31. Flamand, C., Camille Fritzell, Pauline Obale, Quenel, P., \& Raude, J. (2017). The Role of Risk Proximity in the Beliefs and Behaviors Related to Mosquito-Borne Diseases: The Case of Chikungunya in French Guiana. The American journal of tropical medicine and hygiene, 97(2), 344-355. https://doi.org/10.4269/ajtmh.16-1028

\section{Tables}

Due to technical limitations, tables are only available as a download in the supplemental files section.

\section{Figures}



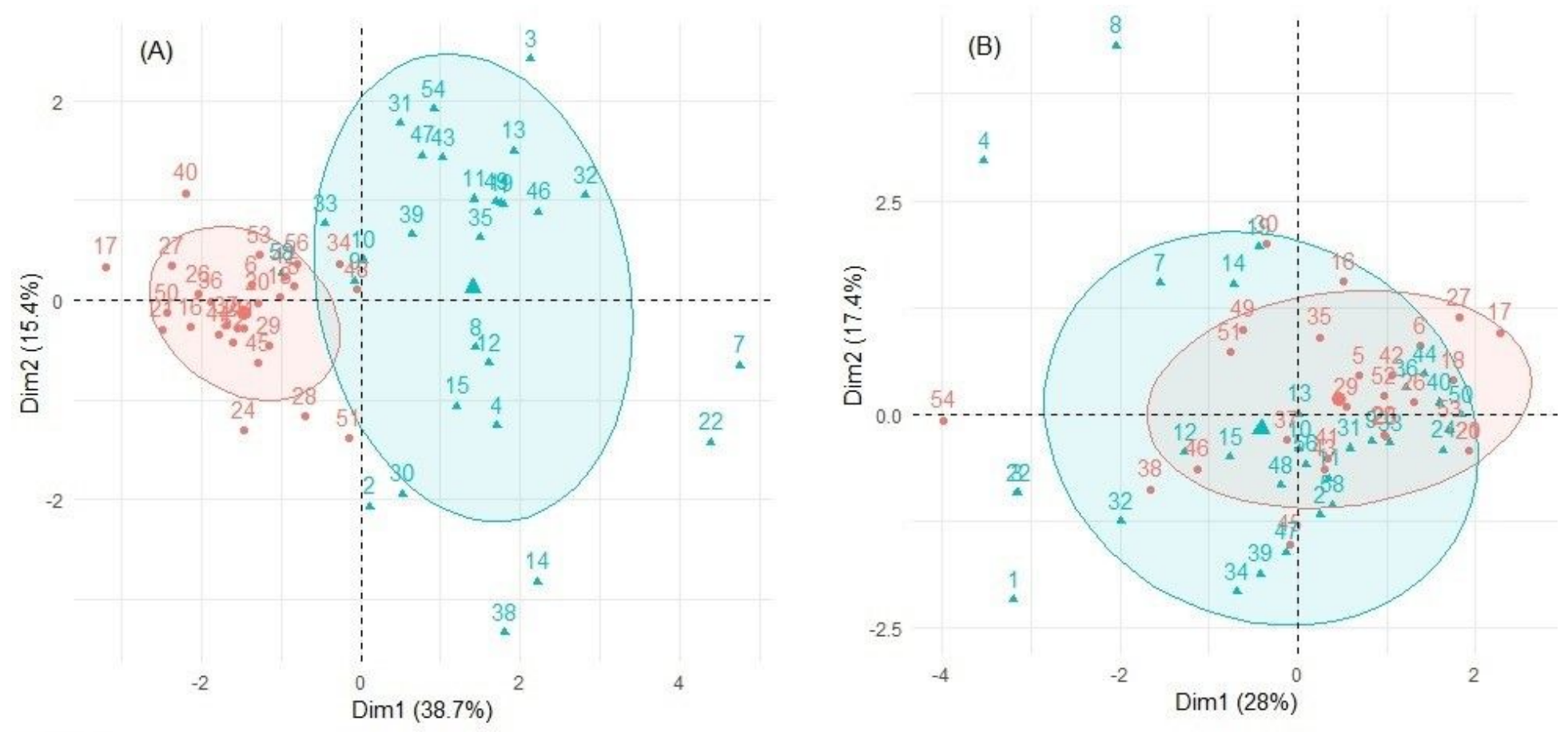

Groups

- Endemic Disease Control Agents (ACEs)

4 Students

\section{Figure 1}

Multivariate analysis showing Biplot plots for individuals and groups (college students and endemic disease control agents - ACEs) with their respective confidence ellipses (with 95\% reliability). Graph A refers to the pre-intervention data analysis and $B$ refers to the post-intervention for arboviruses prevention.

\section{Supplementary Files}

This is a list of supplementary files associated with this preprint. Click to download.

- Table1.docx

- Table5.docx

- Table4.docx

- Table6.docx

- Table2.docx

- Table3.docx 